\title{
Alpha-lipoic acid: mechanisms of action and beneficial effects in the prevention and treatment of diabetic complications
}

\begin{abstract}
Alpha-lipoic acid (ALA) has been widely prescribed drug for treatment and prevention of diabetic complications since it affects the main pathogenesis links. ALA has many biochemical functions acting as a biological antioxidant, anti-inflammatory properties, metal chelators, reducing the oxidized forms of other antioxidant agents such as vitamin $\mathrm{C}$ and $\mathrm{E}$ and glutathione, and modulating the signaling transduction of several pathways, like insulin and nuclear factor kappa-light-chain-enhancer of activated B cells (NF-kB). ALA can protect albumin from glycation, improves the redox state of the plasma and endothelium-dependent vasodilation; induces protein kinase B phosphorylation in vascular endothelial cells; shows a protective effect on oxidative stress-induced apoptosis. In skeletal muscle ALA reduce triglyceride accumulation, enhances expression of the insulin receptor substrate 1 protein and improves insulin sensitivity by activating 5'-AMP-activated protein kinase, recruits glucose transporter type 4 from its storage site in the Golgi to the sarcolemma. Prescription of ALA can have both detrimental and cytoprotective effects on pancreatic $\beta$-cells.
\end{abstract}

Keywords: alpha-lipoic acid, oxidative stress, inflammation, diabetes mellitus, diabetic neuropathy

\section{Introduction}

Diabetes mellitus (DM) is considered as a global public health problem worldwide. The development of cardiovascular and nervous complications is a complex pathological process with a pivotal role of oxidative stress (OS) caused by reactive oxygen species (ROS) and reactive nitrogen species, that is strongly associated with insulin resistance (IR) and DM. ${ }^{1-3}$ The manifestation of DM is accompanied by impaired activity of endothelial nitric oxide synthase (eNOS) and increase of ROS production, thus resulting in increased OS and diminished nitric oxide (NO) bioavailability. ${ }^{4}$ The prevalence of neuropathy in diabetic patients is about $30 \%$, whereas up to $50 \%$ of patients will certainly develop neuropathy during the disease. ${ }^{5,6}$

\section{Discussion}

OS is considered as a main pathophysiological pathway of diabetic neuropathy (DN) development. Increased level of intracellular glucose leads to activation of polyol pathway and formation of advanced glycation end-products (AGE's), resulting in the subsequent formation of ROS. ${ }^{7}$ In the development of DN are involved the following mechanisms: 1. Fructose and sorbitol accumulation due to increased flux through the polyol pathway that is associated with compensatory depletion of other organic osmolytes such as taurine and myo-inositol. 2. Endoneurial microvascular damage and hypoxia due to NO inactivation. Nitric oxide is a signaling molecule with cellular effects, cardiovascular and metabolic process involved in much biological regulation such as cell proliferation, neurotransmission, antimicrobial defence, vasodilatation and inflammatory response. Myeloperoxidase and NO affect the process of inflammation by molecular mechanisms and reaction between them is a sign of local inflammation and cardiovascular events initiation and progression in the body. ${ }^{8,9} 3$. Accumulation of AGE's and subsequent activation of nuclear factor kappa-light-chain-enhancer of activated B cells (NF$\kappa B)$. 4. Homocysteinemia. 5. Increased nerve lipid peroxidation
Volume 7 Issue 4 - 2018

\section{Victoria Serhiyenko, Ludmila Serhiyenko, Galyna Suslik,Alexandr Serhiyenko}

Department of Endocrinology, Danylo Galytsky Lviv National Medical University, Ukraine

Correspondence: Victoria Serhiyenko, Department of Endocrinology, Danylo Halytsky Lviv National Medical University, Ukraine, 69, Pekarska str., 79010, Ukraine, Tel +38 32276949 6, Email 965554@ukr.net, serhiyenkoa@gmail.com

Received: May 15, 2018 | Published: July 09, 2018
(LPO). 6. Modulation of mitogen- activated protein kinases (MAPK's). 7. Abnormal $\mathrm{Ca}^{2+}$ homeostasis and signaling. 8. Decreased expression and level of neurotrophic factors, such as nerve growth factor, neurotrophin 3, and insulin-like growth factor as well as alterations in axonal transport. Given the role of OS in the progression of diabetic peripheral neuropathy (DPN) antioxidants such as alpha lipoic acid (ALA), taurine and acetyl-L-carnitine have been proven to be effective in preventing or delaying the onset of DPN. ${ }^{7,6,10-13 \text {, }}$

Alpha-lipoic acid is a cofactor for $\alpha$-ketoglutarate dehydrogenase and pyruvate dehydrogenase activity, is required for the oxidative decarboxylation of pyruvate to acetyl coenzyme A, which has links citric acid cycle and glycolysis. Both ALA and dihydrolipoic acid (DHLA) are considered as potent antioxidants with the ability to regenerating other factors such as vitamins $\mathrm{C}$ and $\mathrm{E}$ in addition to raising glutathione (GSH) intracellularly. ${ }^{12}$ The following ways are thought to explain the beneficial effects of ALA in reducing the ageassociated alterations in GSH: 1. Impossible delivery of exogenous GSH to tissues such as the brain and heart. 2. The bioavailability of cysteine delivery agents (e.g., $N$-acetylcysteine) is low. However, ALA can modulate the age-related alteration in GSH levels as it is easily taken up into neural tissues. ${ }^{12,14}$ It was suggested that ALA provides its antioxidant effect especially through GSH. Some in vivo and in vitro experimental trials showed that ALA administration increased the intracellular GSH level by $30-70 \% .{ }^{15}$ ALA antioxidant properties in vitro can be explained in different ways: 1 . The concentration of ALA by conduction cell culture studies is often several folds higher than what has been seen in tissues or plasma after a per oral administration. 2. DHLA and ALA are cleared from the culture media slower while in the body $98 \%$ of radiolabeled ALA is excreted in the urine within $24 \mathrm{hrs}^{16}$

Nowadays it is believed that ALA/DHLA are acting as metal chelators and biological antioxidants, modulating the signaling transduction of several pathways, like nuclear factor kappa-light- 
chain-enhancer of activated B cells (NF-kB) and insulin, reducing the oxidized forms of other antioxidant agents (vitamin $\mathrm{E}, \mathrm{C}$ and GSH). Both ALA and DHLA prevent protein carbonyl formation and DHLA alone can regenerate the oxidized forms of vitamins E, $\mathrm{C}$ and to neutralize free radicals. ${ }^{12,17}$ DHLA reduces the oxidized form of ubiquinone $(\mathrm{CoQ} 10)$, which can additionally reduce the alpha-tocopheroxyl radical. ${ }^{12,17,18}$ Alpha-lipoic acid is a pleiotropic medication with anti-inflammatory and antioxidant properties, of which the effects are exerted through the modulation of NF-kB. In fact NF-kB modulates inflammatory cytokines, including interleukin (IL) IL-1 $\beta$ and IL- 6 , in different cell types and tissues. IL-1 $\beta$ and IL-6 undergo DNA methylation-dependent modulation in neural models and pave the road to study the epigenetic mechanisms triggered by ALA. ${ }^{19}$

It is shown that ALA inhibits of nuclear factor of kappa light polypeptide gene enhancer in B-cells inhibitor (I $\mathrm{B}$ ) degradation and NF-kB-dependent gene expression by inhibition of small-molecule inhibitors of IкB kinase 2 (IKK2), suggesting that ALA inhibits NF$\kappa \mathrm{B}$ activation independent of its antioxidant function. ALA inhibits NF- $\kappa$ B activation at the level of or upstream of, IKK- $\alpha$ and IKK- $\beta$, by modulating the mammalian serine/threonine kinase (MEK) MEKK1MKK4-IKK pathway. ${ }^{12,20}$ Alpha-lipoic acid can significantly increase the cellular capacity of GSH synthesis by inducing of nuclear factor erythroid 2-related factor 2 (Nrf-2)-mediated antioxidant gene expression. ALA activates 5'-AMP-activated protein kinase (AMPK) and inhibits NF- $\kappa B$, which in turn have a plethora of metabolic consequences. ALA can modulate peroxisome proliferator activated receptors (PPARs)-regulated genes by activating both PPAR- $\alpha$ and $-\gamma$. PPAR- $\alpha$ regulates the expression of acetyl-CoA synthase and carnitine palmitoyltransferase 1A and PPAR- $\gamma$ increases the expression of lipoprotein lipase, adipocyte fatty acid binding protein and fatty acid translocase/CD36. ${ }^{21,12}$ ALA may increase Nrf2-dependent transcriptional activity by forming lipoyl-cysteinyl mixed disulfides on Kelch-like ECH-associated protein 1 (Keap1), the protein that sequesters $\mathrm{Nrf} 2$ and bridges it to ubiquitin ligases.. ${ }^{22,23}$ Activated protein kinase $\mathrm{C}$ (PKC) increase expression of vascular endothelial growth factor, endothelin, transforming growth factor- $\beta$, plasminogen activator inhibitor- $1, \mathrm{NF \kappa B}$ and the reduced form of nicotinamide adenine dinucleotide phosphate $(\mathrm{NAD}(\mathrm{P}) \mathrm{H})$ oxidases and decrease expression of eNOS. ${ }^{12}$ ALA may directly activate PKC in a redoxactive manner, but it has been shown that $\mathrm{PKC} \delta$ is activated in response to ALA during a type-II transmebrane protein that belongs to the tumor necrosis factor (TNF) family (Fas)-mediated apoptosis. In addition to PKC $\delta$, ALA activates extracellular signal-regulated kinase 1/2 (Erk1/2), p38 MAPK, phosphatidylinositol-4,5-bisphosphate 3 (PI3)-kinase, and protein kinase B (Akt)..$^{24,25}$

Alpha-lipoic acid is effective in reducing OS by preventing LPO and protein damage as other antioxidants significantly inhibit the ROS such as proxy nitrite, NO, hydroxyl radicals, superoxide anion in the membrane and aquatic environment. ${ }^{9}$ Antioxidant action of ALA is mediated by two essential nuclear factors: Nrf2 and NF-kB. ${ }^{19}$ ALA is a powerful lipophilic free radical scavenger of peripheral nerve both in vitro and in vivo. As DM has been associated with increased production and decreased clearance of ROS, OS has been suggested to contribute to defective nerve blood supply and endoneurial oxidative damage. ${ }^{11,26,27}$ Results of some studies show that ALA lowers expression of vascular cell adhesion molecule-1 (VCAM-1) and endothelial adhesion of human monocytes, and inhibits NF- $\mathrm{kB}-$ dependent expression of metalloproteinase-9 in vitro. Similarly, ALA prevents the upregulation of intercellular adhesion molecule-1 and
VCAM-1 in spinal cords and TNF-alpha stimulated cultured brain endothelial cells. ${ }^{23,28}$ ALA is increasingly proposed as an adjuvant in the treatment of many diseases by its pleiotropic properties that allow it to act on different fronts. The conditions that respond to treatment with ALA are characterized by the presence of acclaimed OS or an altered biochemical and molecular mechanism (gene transcription, regulation of enzymatic or receptor activity), ALA can regulate that. Ultimately, diseases with different aetiologies (for example, metabolic syndrome, obesity, DM, multiple sclerosis, heavy metal poisoning, etc.) or physiological conditions such as ageing could benefit from common treatment with ALA. ${ }^{29} \mathrm{OS}$ is associated with hyperglycaemia, and the existence of other triggers factors are supposed to phosphorylate IkB resulting in its degradation and activation of NF-kB which induce the transcription of several molecules related to the migration of monocytes, vascular adhesion and inflammation. The inhibition of NF-kB by ALA is probably related to its possibility to decrease the IkB degradation through modulation of upstream kinases like MAPK or to inhibit PKC which is also able to phosphorylate $\mathrm{IkB} .{ }^{30}$

ALA has drawn considerable attention for treatment and prevention of chronic DM complications. ${ }^{4}$ ALA supplementation is an effective strategy for mitochondrial function in chronic inflammatory states; increased mitochondrial capacity, partially restored mitochondrial enzyme activity and increased ATP production, when in the presence of inflammatory induced NO. Other research also shows small molecular S-nitosothiols, which act as reservoirs and donors for NO, downregulate mitochondrial function. The pro-oxidant action of ALA/ DHLA is not fully understood but could be related to different direct or indirect reactions such as oxidation of DHLA by ubisemiquinone, to the maintenance of NrF2 into the cytosol through a linkage to Keap1 protein, resulting in the inhibition of the transcription of cytoprotective genes which include many antioxidant genes or to an ubiquination of Nrf2 in a Keap1-dependent action. Considering the latter action, ALA/DHLA is an activator/inducer of translocation of Nrf2 to the nucleus for regulation of antioxidant gene expression. ${ }^{30-32}$ ALA had a protective effect on mitochondrial damage and neurotoxicity caused by chemotherapeutic agents; has protective activity on nervous system lesions caused by chronic constriction injury of the peripheral nerve; topical administration of ALA in a transaction model of sciatic nerve provided faster healing; ALA that was administered $3 \mathrm{~d}$ before ischemia resulted in a significant protective effect from moderate ischemia-reperfusion injury of the peripheral nerve. ${ }^{15}$

The regulatory functions of ALA may now include altering protein S-nitrosylation and regulating the expression of some proteins affected by S-nitrosylation mechanisms. GSH levels were also analyzed and it is proposed that this new mechanism of ALA may be through the regulation of $\mathrm{GSH}$, as this antioxidant protects mitochondrial complexes from NO-induced damage. The diminished mitochondrial enzyme function central to energy production, due to nitrosative stress, can be alleviated with ALA supplementation through S-nitrosylation regulation. When ALA was introduced, it significantly reduced S-nitrosylation in both of these mitochondrial enzyme complexes and effectively restored mitochondrial enzyme activities inhibited by excess NO, while significantly increasing ATP production. ${ }^{33-35}$ Alpha-lipoic acid has shown considerable promise as an antiosteoclastogenic agent due to its potent ROS-scavenging capabilities along with a proven clinical safety record. Collectively, current data indicate that ALA protects from bone loss via a whopronged mechanism involving inhibition of osteoclastogenic ROS generation and upregulation of redox gene expression. ${ }^{36}$ 
ALA/DHLA are considered as chelator compounds, in particular ALA chelates mostly the $\mathrm{Cu}^{2+}, \mathrm{Mn}^{2+}, \mathrm{Pb}^{2+}$ and $\mathrm{Zn}^{2+}$ and DHLA, in addition, is also able to chelate $\mathrm{Fe}^{3+}$ and $\mathrm{Hg}^{2+}$. The administration of ALA was followed by decreased iron uptake and its diminished cytosolic reactive pool in cultured lens epithelial cells that was associated with increased cell resistance to a $\mathrm{H}_{2} \mathrm{O}_{2}$ challenge and reduction of the iron-induced OS. ${ }^{12,15,30}$ ALA treatment of immortalized mouse myoblast cell line $(\mathrm{C} 2 \mathrm{C} 12)$ myotubes increased the intracellular $\mathrm{Ca}^{2+}$ concentration, suggesting that ALA may activate AMPK by stimulating the calmodulin-dependent protein kinase kinase (CaMKK) pathway. ${ }^{12}$ Another potential benefit of using ALA in the treatment of DM complications is connected with glycation reactions inhibition. Both ALA and DHLA can protect albumin from glycation, in particular ALA reduces protein glycosylation, lower LPO, and increase $\mathrm{Na}^{+} / \mathrm{K}^{+}$-ATPase activity in human red blood cells exposed to high glucose concentration. The effects of ALA on DN have also been studied in streptozotocin-induced diabetic rats, where ALA promoted glucose uptake by nerve cells, increased nerve myoinositol, GSH levels, $\mathrm{Na}^{+} / \mathrm{K}^{+}$-ATPase activity, and nerve blood flow while normalizing NAD/NADH ratios. ${ }^{12,37}$

ALA improves the redox state of the plasma and endotheliumdependent vasodilation. ALA also induces Akt phosphorylation in human umbilical vascular endothelial cells and the human monocytic leukemia cell line-1. These studies suggest that improved endothelial function due to ALA is at least partially attributed to recoupling of eNOS and increased NO bioavailability. ${ }^{12,38,39}$ ALA was shown to reduce asymmetric dimethylarginine in diabetic patients with end-stage renal disease and with normal renal function. These data provide a novel mechanism by which ALA regulates endothelial function. ${ }^{12,40,41}$ Another possible mechanism of ALA's protective effect may be decrease of apoptosis. Apoptosis plays an important role in the death of the cell body of injured axons, Schwann cells, phagocytic neutrophils and macrophages after peripheral nerve injury. The incidence of apoptosis-related cell death in dorsal root ganglion neurons following axonotmesis ranges from 20 to $50 \%$. Death occurs more frequently when axonotmesis occurs it was shown that ALA causes a down-regulation of NF- $\mathrm{KB}$ that plays a fundamental role in the expression of various genes that are involved in the inflammatory response and also that are involved in cell apoptosis processes. Also, it was shown that ALA pre-treatment reduced radiation-induced apoptotic and necrotic cell death of granule cells and Purkinje cells in two experimental studies. Acute treatments with ALA were shown to cause to both reduction of expression of NF- $\kappa B$ and matrix metalloproteinase-9, an enzyme responsible for the degradation of the extracellular matrix. ${ }^{15,42-44}$

ALA was also found to enhance expression of the insulin receptor substrate 1 (IRS1) protein in muscle of obese Zucker rats, and it also elicited association of IRS1 with the p85 regulatory subunit of PI3K. In skeletal muscle, ALA is proposed to recruit glucose transporter type 4 (GLUT4) from its storage site in the Golgi to the sarcolemma, so that glucose uptake is stimulated by the local increase in transporter abundance. Evidence from cell culture experiments supports the involvement of the insulin-signaling cascade in ALA-stimulated translocation of GLUT1 and GLUT4. ${ }^{23,45,46}$ Improvement of insulin sensitivity induced by ALA is mediated by AMPK activation and reduction of triglyceride accumulation in skeletal muscle. The cytoprotective or detrimental effects of ALA on pancreatic $\beta$-cells depends on the concentration of ALA and underlying pathophysiologic state. It is considered that at high concentations ALA is detrimental to $\beta$-cells, but at clinically relevant concentrations exerts cytoprotective effects. ${ }^{12}$ ALA has many actions in the glucose uptake, glycogen synthesis and insulin metabolic pathways with some differences between both isomers. The results of some studies performed in vitro have proved that R-ALA increases the translocation of GLUT4 and GLUT1 to the skeletal muscle cells and plasmatic membrane of adipocytes kept in a culture milieu. ${ }^{30,47}$ ALA has some important functions in the AMPK expression and activity in the brain and peripheral tissues. AMPK is involved in many intracellular pathways related to cell cycle, stress response, metabolism and ageing. ALA could increase the activation of AMPK indirectly by activating CaMKK. ALA is also able to modulate the AMPK activity in the brain by metabolic stresses that inhibit ATP production such as OS, glucose deprivation, hypoxia and ischemia. ${ }^{30,48}$

\section{Conclusion}

The many unique properties of ALA, namely antioxidant effects, inhibition of glycation reactions, prevention of beta-cell destruction, enhancement of glucose uptake, restoration of vitamins levels (such as vitamin $\mathrm{E}$ and $\mathrm{C}$ ), improvement of neurons function and conduction has been shown in some experimental and clinical trials. ALA due to its essential role in mitochondrial bioenergetic reactions was considered as a valuable therapeutic option in prevention and treatment of chronic diabetes mellitus complications. However, further work with better-designed protocol and appropriate selection of study population, doses and duration may provide evidence for the hidden therapeutic potential of ALA and its potential properties for other diabetic complications such as diabetic autonomic neuropathy.

\section{Acknowledgements}

None.

\section{Conflict of interest}

The authors declare that there is no conflict of interest.

\section{References}

1. American Diabetes Association. Standards of medical care in diabetes 2017. Diabetes Care. 2017;40(1S):S1-S132.

2. Pitocco D, Tesauro M, Alessandro R, et al. Oxidative stress in diabetes: implications for vascular and other complications. Int $J \mathrm{Mol} \mathrm{Sci}$. 2013;14(11):21525-21550.

3. Serhiyenko VA, Serhiyenko AA. Cardiac autonomic neuropathy: Risk factors, diagnosis and treatment. World J Diabetes. 2018;9(1):1-24.

4. Rochette L, Ghibu S, Muresan A, et al. Alpha-lipoic acid: molecular mechanisms and therapeutic potential in diabetes. Can J Physiol Pharmacol. 2015;93(12):1021-1027.

5. Callaghan BC, Cheng HT, Stables CL, et al. Diabetic neuropathy: Clinical manifestations and current treatment. Lancet Neurol. 2012;11(6):521534 .

6. Boghdadi MA, Afify HE, Sabri N, et al. Comparative study of vitamin B complex combined with alpha lipoic acid versus vitamin B complex in the treatment of diabetic polyneuropathy in type 2 diabetic patients. Clin Exp Pharmacol. 2017;7:241.

7. Hosseini A, Abdollahi M. Diabetic neuropathy and oxidative stress: Therapeutic perspectives. Oxid Med Cell Longe. 2013:1-15.

8. Garça MF, Aslan M, Tuna B, et al. Serum myeloperoxidase activity, total antioxidant capacity and nitric oxide levels in patients with chronic otitis media. J Membr Biol. 2013;246(7):519-524. 
9. Ahmadvand H, Jamor P. Effects of alpha lipoic acid on level of NO and MPO activity in diabetic rats. Ann Res Antioxid. 2017;2(2): E04

10. Ziegler D, Low PA, Litchy WJ. Efficacy and safety of antioxidant treatment with $\alpha$-lipoic acid over 4 years in diabetic polyneuropathy: the NATHAN 1 trial. Diabetes Care. 2011;34(9):2054-2060.

11. Vallianou N, Evangelopoulos A, Koutalas P. Alpha-lipoic acid and diabetic neuropathy. Rev Diabet Stud. 2009;6(4):230-236.

12. Golbidi S, Badran M, Laher I. Diabetes and alpha lipoic acid. Front Pharmacol. 2011;2:69.

13. Shakher J, Stevens MJ. Update on the management of diabetic polyneuropathies. Diabetes Metab Syndr Obes. 2011;4:289-305.

14. Suh JH, Wang H, Liu RM, et al. (R)- alpha-lipoic acid reverses the agerelated loss in GSH redox status in post-mitotic tissues: evidence for increased cysteine requirement for GSH synthesis. Arch Biochem Biophys. 2004;423(1):126-135.

15. Kocaoğlu S, Aktaş Ö, Zengi O, et al. Effects of alpha lipoic acid on motor function and antioxidant enzyme activity of nerve tissue after sciatic nerve crush injury in rats. Turk Neurosurg. 2017;1:1-8.

16. Shay KP, Moreau RF, Smith EJ, et al. Alpha-lipoic acid as a dietary supplement: Molecular mechanisms and therapeutic potential. Biochim Biophys Acta. 2009;1790(10):1149-1160.

17. Bast A, Haenen GR. Lipoic acid: a multifunctional antioxidant Biofactors. 2003;17(1-4):207-213

18. Smith AR, Hagen TM. Vascular endothelial dysfunction in aging: los of Akt-dependent endothelial nitric oxide synthase phosphorylation and partial restoration by (R)-alpha-lipoic acid. Biochem Soc Trans. 2003;31(Pt6):1447-1449.

19. Dinicola S, Proietti S, Cucina A, et al. Alpha-lipoic acid downregulates IL-1 $\beta$ and IL-6 by DNA hypermethylation in SK-N-BE neuroblastoma cells. Antioxidants (Basel). 2017;6(4):E74.

20. Ying Z, Kampfrath T, Sun Q, et al. Evidence that $\alpha$-lipoic acid inhibits NFK-B activation independent of its antioxidant function. Inflamm Res. 2011;60(3):219-225.

21. Butler JA, Hagen TM, Moreau R. Lipoic acid improves hypertriglyceridemia by stimulating triacylglycerol clearance and downregulating liver triacylglycerol secretion. Arch Biochem Biophys. 2009;485(1):63-71.

22. Yamamoto T, Suzuki T, Kobayashi A, et al. Physiological significance of reactive cysteine residues of Keap1 in determining Nrf2 activity. Mol Cell Biol. 2008;28(8):2758-2770.

23. Shay K, Hagen TM. Age-associated impairment of Akt phosphorylation in primary rat hepatocytes is remediated by alpha-lipoic acid through PI3 kinase, PTEN, and PP2A. Biogerontology. 2009;10(4):443-456.

24. Kim YS, Podder B, Song HY. Cytoprotective effect of alpha-lipoic acid on paraquat-exposed human bronchial epithelial cells via activation of nuclear factor erythroid related factor-2 pathway. Biol Pharm Bull. 2013;36(5):802-811.

25. Monastra G, De Grazia S, Cilaker Micili S, et al. Immunomodulatory activities of alpha lipoic acid with a special focus on its efficacy in preventing miscarriage. Expert Opin Drug Deliv. 2016;13(12):16951708.

26. Kozlov AV, Gille L, Staniek K, et al. Dihydrolipoic acid maintains ubiquinone in the antioxidant active form by two-electron reduction of ubiquinone and one electron reduction of ubisemiquinone. Arch Biochem Biophys. 1999;363(1):148-154

27. Chaudhary P, Marracci GH, Bourdette DN. Lipoic acid inhibits expression of ICAM-1 and VCAM-1 by CNS endothelial cells and T cell migration into the spinal cord in experimental autoimmune encephalomyelitis. $J$ Neuroimmunol. 2006;175(1-2):87-96

28. Packer L, Cadenas E. Lipoic acid: energy metabolism and redox regulation of transcription and cell signalling. $J$ Clin Biochem Nutr. 2011;48(1):26-32.

29. Maglione E, Marrese C, Migliaro E, et al. Increasing bioavailability of (R)-alpha-lipoic acid to boost antioxidant activity in the treatment of neuropathic pain. Acta Biomed. 2015;86(3):226-233.

30. Gomes MB, Negrato CA. Alpha-lipoic acid a pleiotropic compound with potential therapeutic use in diabetes and other chronic diseases. Diabetol Metab Syndr. 2014;6(1):80.

31. Packer L, Kraemer K, Rimbach G. Molecular aspects of lipoic acid in the prevention of diabetes complications. Nutrition. 2001;17(10): 888-895.

32. Koriyama Y, Nakayama Y, Matsugo S, et al. Protective effect of lipoic acid against oxidative stress is mediated by Keap1/Nrf2-dependent heme oxygenase-1 induction in the RGC-5 cellline. Brain Res. 2013;1499:145157

33. Rouchette L, Ghibu S, Richard C, et al. Direct and indirect antioxidan properties of $\alpha$-lipoic acid. Mol Nutr Food Res. 2013;57(1):114-125.

34. Park S, Karunakaran U, Ho Jeoumg N, et al. Physiological effect and therapeutic application of alpha lipoic acid. Curr Med Chem. 2014;21(32):3636-3645

35. Hiller S, DeKroon R, Hamlett ED, et al. Alpha-lipoic acid supplementation protects enzymes from damage by nitrosative and oxidative stress. Biochim Biophys Acta. 2016;1860(1PtA):36-45.

36. Roberts JL, Moreau R. Emerging role of alpha-lipoic acid in the prevention and treatment of bone loss. Nutr Rev. 2015;73(2):116-125.

37. Stuhlinger MC, Abbasi F, Chu JW, et al. Relationship between insulin resistance and an endogenous nitric oxide synthase inhibitor. JAMA. 2002;287(11):1420-1426

38. Zhang WJ, Wei H, Hagen T, et al.Alpha-lipoic acid attenuates LPS-induced inflammatory responses by activating the phosphoinositide 3-kinase/Akt signaling pathway. Proc Natl Acad Sci USA. 2007;104(10):4077-4082.

39. Sena CM, Nunes E, Louro T, et al. Effects of alpha-lipoic acid on endothelial function in aged diabetic and high-fat fed rats. $\mathrm{Br} J$ Pharmacol. 2008;153(6):894-906.

40. Chang JW, Lee EK, Kim TH, et al. Effects of alpha-lipoic acid on the plasma levels of asymmetric dimethylarginine in diabetic end-stage renal disease patients on hemodialysis: A pilot study. Am J Nephrol. 2007;27(1):70-74

41. Mittermayer F, Pleiner J, Francesconi M, et al. Treatment with alphalipoic acid reduces asymmetric dimethylarginine in patients with type 2 diabetes mellitus. Transl Res. 2010;155(1):6-9.

42. Tomassoni D, Amenta F, Di Cesare Mannelli L, et al. Neuroprotective activity of thioctic acid in central nervous system lesions consequent to peripheral nerve injury. BioMed Res Int. 2013;2013:985093.

43. Azizi S, Heshmatian B, Amini K, et al. Alpha lipoic acid loaded in chitosan conduit enhances sciatic nerve regeneration in rat. Iran J Basic Med Sci. 2015;18(3):228-233.

44. Constantino M, Guaraldi C, Constantino D, et al. Peripheral neuropathy in obstetrics: Efficacy and safety of $\alpha$-lipoic acid supplementation. Eur Rew Med Pharmacol Sci. 2014;18(18):2766-2771.

45. Foley TD, Petro LA, Stredny CM, et al. Oxidative inhibition of protein phosphatase 2A activity: role of catalytic subunit disulfides. Neurochem Res. 2007;32(11):1957-1964.

46. Ross SH, Lindsay Y, Safrany ST, et al. Differential redox regulation within the PTP superfamily. Cell Signal. 2007;19(7):1521-1530. 
47. Wang Y, Li X, Guo Y, et al. Alpha-Lipoic acid increases energy expenditure by enhancing adenosine monophosphate-activated protein kinase-peroxisome proliferator-activated receptor-gamma coactivatorlalpha signaling in the skeletal muscle of aged mice. Metabolism. 2010;59(7): 967-976.
48. Ramamurthy S, Ronnet G. AMP-activated protein kinase (AMPK) and energy sensing in the brain. Exp Neurobiol. 2012;21(2):52-60 